\title{
HEADING CONTROL SYSTEM DESIGN FOR A MICRO-USV BASED ON AN ADAPTIVE EXPERT S-PID ALGORITHM
}

\author{
Runlong Miao \\ Science and Technology on Underwater Vehicle Laboratory, Harbin Engineering University, China \\ Zaopeng Dong \\ School of Transportation, Wuhan University of Technology, China \\ Lei Wan \\ Science and Technology on Underwater Vehicle Laboratory, Harbin Engineering University, China \\ Jiangfeng Zeng \\ Science and Technology on Underwater Vehicle Laboratory, Harbin Engineering University, China
}

\begin{abstract}
The process of heading control system design for a kind of micro-unmanned surface vessel (micro-USV) is addressed in this paper and a novel adaptive expert S-PID algorithm is proposed. First, a motion control system for the micro-USV is designed based on STM32-ARM and the PC monitoring system is developed based on Labwindows/CVI. Second, by combining the expert control technology, S plane and PID control algorithms, an adaptive expert S-PID control algorithm is proposed for heading control of the micro-USV. Third, based on SL micro-USV developed in this paper, a large number of pool experiments and lake experiments are carried out, to verify the effectiveness and reliability of the motion control system designed and the heading control algorithm proposed. A great amount of comparative experiment results shows the superiority of the proposed adaptive expert S-PID algorithm in terms of heading control of the SL micro-USV.
\end{abstract}

Keywords: micro unmanned surface vessel (micro-USV); control system design; adaptive expert S-PID algorithm; heading control; pool experiment; lake experiment

\section{INTRODUCTION}

Unmanned Surface Vessels (USVs) are attracting growing attention from researchers all over the world because of their extensive applications in military reconnaissance, homeland security, hydrographic surveys, environmental monitoring, marine scientific exploration, coordinating work with Autonomous Underwater Vehicles (AUV) [1-4]. A micro-USV is a kind of smaller unmanned surface vessel, usually less than three meters in length. As micro-USVs have the characteristics of low development cost, convenient transportation, ease to carry out experimental research and so on, at present, many scholars have carried out relevant research work on them. SESAMO micro-USV was developed in 2005 by Caccia $M$. et al and the heading control algorithms are both based on traditional PID, meanwhile several control trials were performed in the Genoa harbor [5]. Do K. D. et al designed a series of observer estimation and way point guidance algorithms and applied them to their KD micro-USV. The path following control is then achieved based on heading and speed control [6]. Fuzzy control technology and PID are combined by Sohn et al. Fuzzy PID was proposed and used in their FC micro-USV, and also some outdoor experiments are carried out to verify the effectiveness of the control system [7]. 
S algorithm is presented by Wu G. X. for their XL USV's heading control - some lake experiments are carried out in Longfengshan reservoirs [8]. Besides, heading control of a ship in the presence of environment disturbances is addressed by Sun in [9], and a model predictive controller with disturbance compensation is designed. By combining cascade system theory, nonlinear backstepping method and PID control technology, heading controller for a USV is designed by Sonnenburg [10]. Using modular design principle, a hybrid PID control algorithm for USV's heading control system is proposed by Kurowsi based on multiple input multiple output (MIMO) system theory and PID control technology [11]. An improved PID control algorithm is proposed based on nonlinear compensation principle by Nad in [12]. But all the research results in [9-12] are achieved in the simulation conditions, no practical experiments are carried out, which is the same situation in literature works [13-17].

According to the above analysis, at present, many researches on heading control of the USV or micro-USV are still in the stage of theoretical analysis and simulation experiments [9-12]. A small number of experiment research results are still based on traditional PID control algorithm and $S$ plane algorithm. In addition, the published research results on micro-USV's system design are relatively small, and the system integration and design ideas are not described clearly, which is of great concern to the system designer.

Motivated by the above considerations, a novel adaptive expert S-PID control algorithm is proposed for heading control of SL micro-USV developed in this paper, and hardware and software design process and system integration process of the micro-USV are introduced in detail which can provide important reference for the relevant researchers. Compared with the existing research results, the main contributions of this paper are as follows: (i) a novel adaptive expert S-PID control algorithm is firstly proposed in this paper, which could significantly enhance the micro-USV's heading control performance compared with traditional $S$ plane motion control algorithm. (ii) Hardware and software design process and system integration process of the microUSV are introduced in detail which can provide important reference for the relevant researchers. (iii) A large number of pool experiments and lake experiments are carried out in this paper, and heading control performance of different control algorithms is compared and analyzed based on these large amounts of experiment results. All these experiment results provide an important reference for the heading control algorithm design of other USVs. (iv) This paper clearly points out the issues that need attention during the USV's motion control system design process, and gives solutions to some common problems. (v) Problems that need attention during the experiment process are put forward, and the solutions to those common problems are given according to the authors practical experience.

\section{SYSTEM DESIGN OF SL MICRO-USV}

SL micro-USV developed in this paper is shown as follows in figure 1:

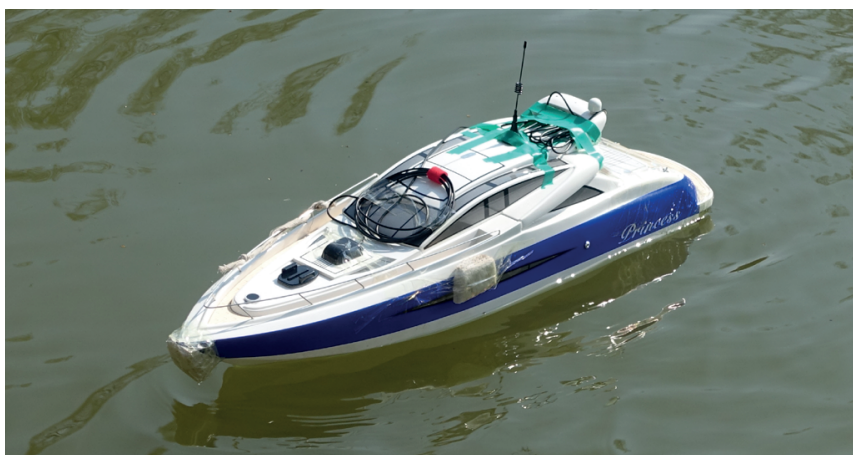

Fig.1. SL micro-USV

SL micro-USV is about 0.98 meters long, 0.25 meters wide, 0.23 meters high (not including the sucker antenna), it weighs about $5 \mathrm{~kg}$ and the hull is made of high strength vinyl resin (fiber glass). It is equipped with a SSS3660/1620kv brushless motor, which is made of top silicon steel sheet, magnetic steel, high performance bearings etc.. The motor has an effective efficiency of up to $90 \%$, it could effectively provide the SL micro-USV surging power and it is driven by the HW-120A brushless electronic speed control (ESC). Besides, the SL micro-USV is equipped with a $4 \mathrm{~mm}$ driven shaft system, a $75 \mathrm{~mm}$ long rudder and a $48 \mathrm{~mm}$ two-leaf copper propeller. SL micro-USV's hardware system design process and software system design process will be described in detail in the next two sections.

\section{HARDWARE SYSTEM DESIGN PROCESS OF SL MICRO-USV}

Bottom control system of SL micro-USV is designed based on STM32F103ZET6-ARM core control board, which has the characteristics of high performance, low cost, low power consumption etc. Compared with a traditional 51 single chip microcomputer, STM32-ARM has faster computing speed, more functions, larger storage space, and it has a rich peripheral interface that can be a convenient connection for different kinds of external devices and sensors. It is equipped with a UBLOX NEO-6M GPS module for obtaining its position and speed information during sailing and also a three dimensional electronic compass DCM260B-Compass for obtaining heading information. With hard magnetic, soft magnetic and inclination compensation functions, the DCM260B-Compass's heading measurement accuracy is up to 0.8 degree. The APC802 wireless communication module is used in the SL micro-USV, to achieve real-time data transmission and communication between the bottom control system and the upper computer monitoring system. The detail motion control system schematic of the SL microUSV is shown below in figure 2 . 


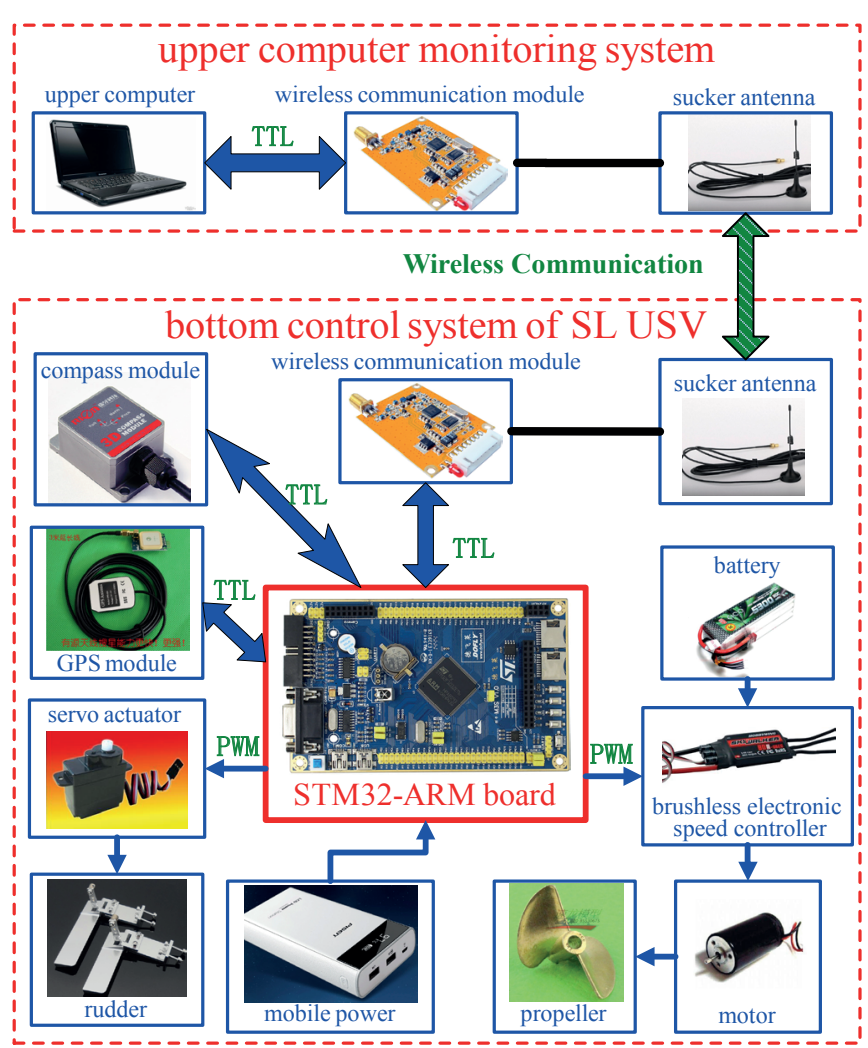

Fig.2. Motion control system schematic of SL micro-USV

In order to show the internal structure and the hardware connection diagram more clearly, figure 3 is given below, where the signal wire 1 is the control signal line of ESC and signal wire 2 is the rudder control signal line. What needs to be noted is that, the control signal of ESC and rudder's servo actuator are both Pulse Width Modulation (PWM) signals. The frequency of the signals need to be accessed manually, or one could obtain them by testing the output signal frequency of the original remote control handle of the ship model. Our test show that the wrong frequency will not start the motor and the ESC has a protection function, one needs to design the output PWM signal in accordance with the ESC's start process requirements.

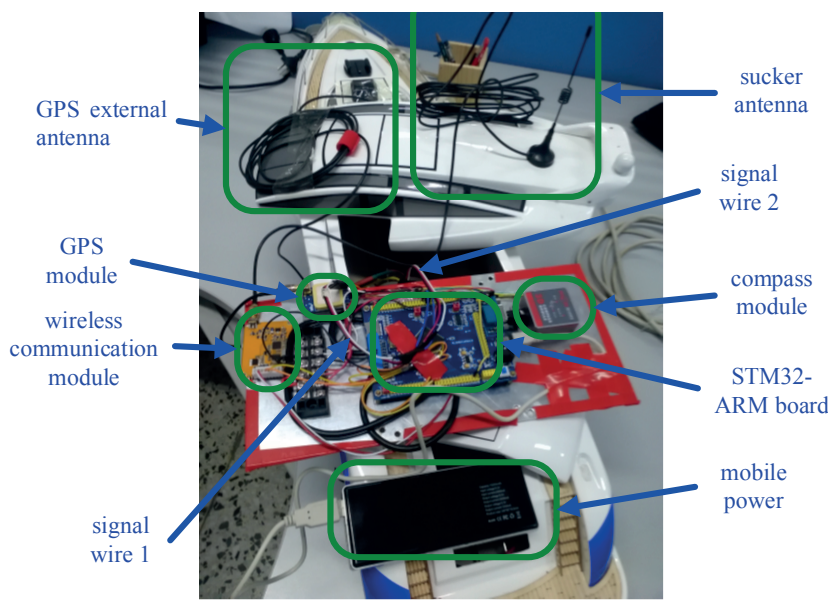

Fig.3. Internal structure and the hardware connection diagram of SL USV

\section{SOFTWARE SYSTEM DESIGN PROGRESS OF SL MICRO-USV}

The SL micro-USV's control system is mainly composed of the upper computer monitoring system and the bottom control system; see as follows in figure 4.

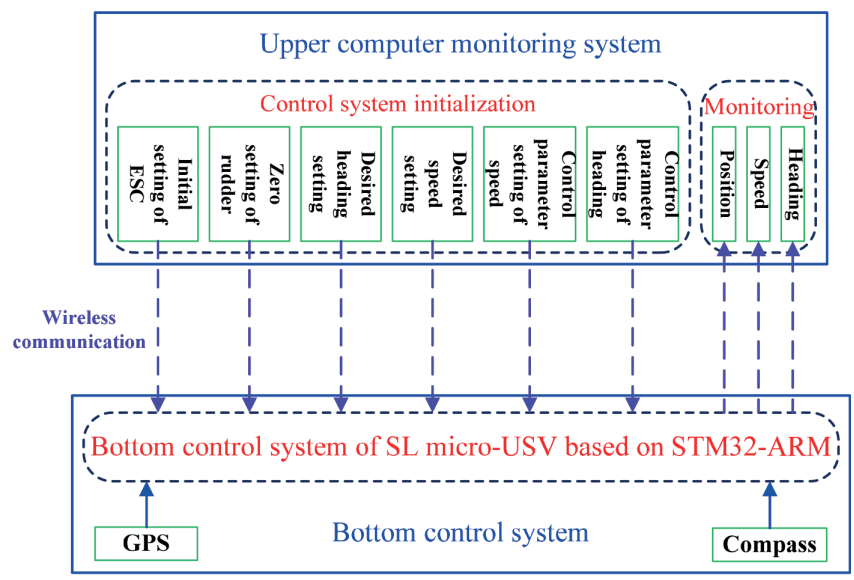

Fig.4. Information interaction diagram of SL USV

The upper computer monitoring system is developed based on Labwindows/CVI, which is a proven ANSI C integrated development environment and engineering toolbox. Upper computer monitoring system of SL USV is shown below in figure 5 .

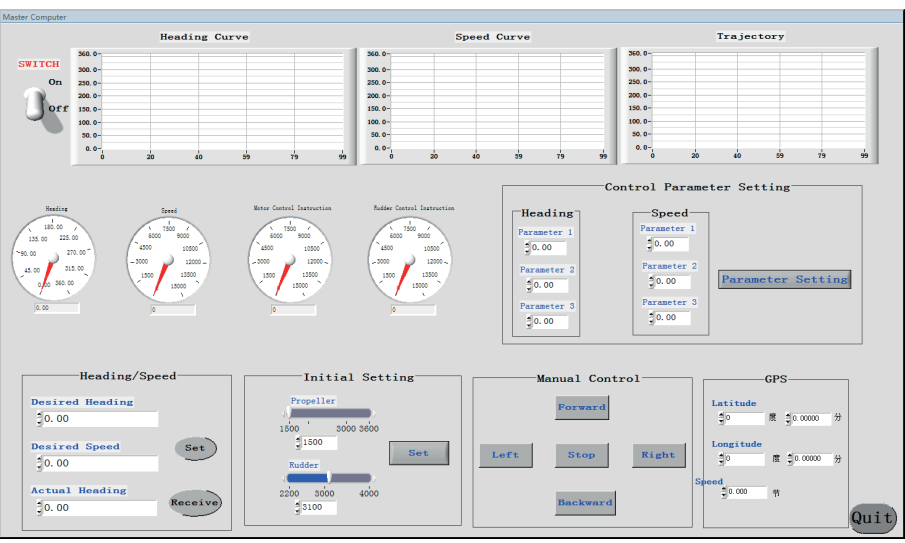

Fig.5. Upper computer monitoring system of SL USV

\section{ADAPTIVE EXPERT S-PID CONTROL ALGORITHM DESIGN}

\section{S PLANE CONTROL ALGORITHM}

The S plane control algorithm is a kind of nonlinear control algorithm derived from fuzzy logic control theory and PID control technology, learning from the mathematical description form of the Sigmoid curve function and Sigmoid surface function. The $S$ plane control algorithm can be described as follows: 


$$
u=\frac{2}{1+\exp \left(-k_{1} e-k_{2} \dot{e}\right)}-1
$$

where $e, \dot{e} 、 u$ are the normalized state error value, state error value change rate, control variable, $k_{1}$ and $k_{2}$ are the controller parameters to be designed. In general, the two control parameters $\left(k_{1}\right.$ and $\left.k_{2}\right)$ of the S plane control algorithm are manually adjusted by the controller designer, in order to achieve the local optimal control under different states. Considering the manual adjusting of the $\mathrm{S}$ plane control algorithm parameters, it is more cumbersome and requires long human intervention. The authors group has accumulated a lot of manual adjustment experience in parameters adjustment of the $\mathrm{S}$ plane control algorithm. The expert control technology is introduced in this paper, and then the large amounts of manual parameter adjustment experience could be described in the form of expert control rules. Which could help to achieve adaptive parameters adjustment. Then it would improve the accuracy and adaptability of the motion control system.

\section{PID CONTROL ALGORITHM}

The PID control algorithm continuously calculates the error value $e(t)$ as the difference between a desired set point and a measured process variable and applies a correction based on proportional, integral, and derivative terms. The controller attempts to minimize the error over time by the adjustment of a control variable $u(t)$, the new value determinate by a weighted sum:

$$
u(t)=k_{p} e(t)+k_{i} \int_{0}^{t} e(t) d t+k_{d} \frac{d e(t)}{d t}
$$

where $k_{p}, k_{i}$ and $k_{d}$, are all non-negative, denote the coefficients for the proportional, integral and derivative terms, respectively (sometimes denoted P, I and D). As PID controller relies only on the measured process variable, not on knowledge of the underlying process, it is broadly applicable.

\section{EXPERT CONTROL SYSTEM}

In artificial intelligence, an expert control system is designed to solve complex problems by reasoning based on knowledge, represented mainly as if-then rules rather than through conventional procedural code. An expert control system is divided into two subsystems: the inference engine and the knowledge base. The knowledge represents facts and rules. The inference engine applies the rules to the known facts to deduce new facts. Inference engines can also include explanation and debugging abilities. A typical expert control system block diagram is shown below:

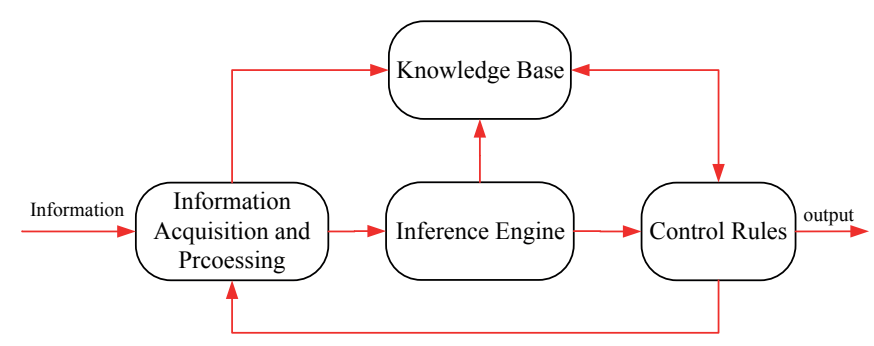

Fig.6. Expert control system block diagram

\section{ADAPTIVE EXPERT S-PID CONTROL ALGORITHM}

The traditional PID control algorithm is simple and easy to implement, but it is a typical linear control algorithm, the application of nonlinear systems is limited. In order to solve the nonlinear problem of the USV's heading control system, a nonlinear $\mathrm{S}$ surface algorithm is integrated into the controller design process. In addition to considering the control parameters of $S$ algorithm, it needs to be constantly adjusted to adapt to different nonlinear characteristics under different environment conditions. An adaptive expert controller is proposed to optimize the control parameters of the $\mathrm{S}$ algorithm, and then an adaptive Expert S-PID control algorithm is designed as follows:

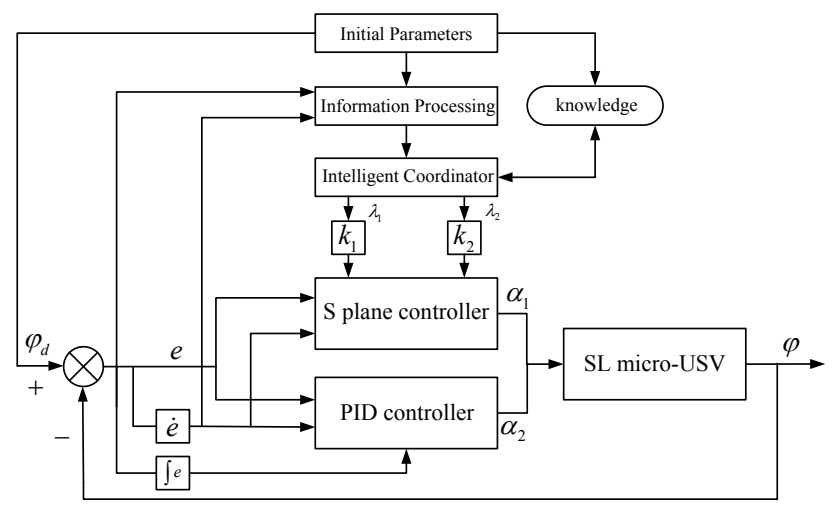

Fig.7. Adaptive Expert S-PID control system of SL USV

In figure 7 , initial parameters mainly include desired heading angle, threshold values of heading angle deviation and its differentiation, initial control parameters of $S$ algorithm and PID control algorithm, dynamic correction factor of S algorithm's control parameters etc.

Information processing, mainly includes acquisition and processing of USV's current heading angle, heading angle deviation and its differentiation etc., USV's control system, USV's PC monitoring system.

The knowledge which the authors group has accumulated is based on a large number of artificial parameters adjustment experience in heading control of unmanned marine vehicles using $\mathrm{S}$ algorithm. Combined with the initial parameters, threshold values of heading angle deviation and its differentiation, initial control parameters of $S$ algorithm and 
dynamic correction factor of S algorithm's control parameters, the expert control rules are developed and then the intelligent coordinator acts on them.

Intelligent coordinator is based on the empirical knowledge of the control system, through the reasoning mechanism, to solve the operation and produce the basic control parameters' online adjustment method, and at the same time to modify the control strategy.

According to a large amount of pool experiments, lake experiments and sea experiments carried out by the authors group, the greater the value of $k_{1}$ and $k_{2}$, the higher the response sensitivity of the controller to the deviation and its differentiation, accompanied by a more pronounced overshoot and oscillation. On the other hand, the smaller the control parameter value, the weaker the overshoot and oscillation of the controller and relatively slower response of the control system to the deviation and its differentiation. Normally, the initial value of the control parameters of $k_{1}$ and $k_{2}$ are both chosen to be about 3.0. If the overshoot is too large, parameter $k_{1}$ can be slightly reduced with an appropriate increase of parameter $k_{2}$. Conversely, if the convergence rate is too slow, one can increase the parameter $k_{1}$ appropriately and reduce the parameter $k_{2}$ slightly, in order to achieve local optimal control. Based on the above experience, control rules can be designed as follows:

Rule 1 If $e>d_{1} \cap \dot{e}>d_{2}$, then $\lambda_{1}=0, \lambda_{2}=0$;

Rule 2 If $e>d_{1} \cap e>0 \cap \dot{e}<d_{2}$, then $\lambda_{1}=0, \lambda_{2}=\Delta_{2}$;

Rule 3 If $e>d_{1} \cap e<0 \cap \dot{e}>-d_{2}$, then $\lambda_{1}=\Delta_{1}, \lambda_{2}=\Delta_{2}$;

Rule 4 If $e>d_{1} \cap \dot{e}<-d_{2}$, then $\lambda_{1}=0, \lambda_{2}=-\Delta_{2}$;

Rule 5 If $e>0 \cap e<d_{1} \cap \dot{e}>d_{2}$, then $\lambda_{1}=\Delta_{1}, \lambda_{2}=0$;

Rule 6 If $e>0 \cap e<d_{1} \cap \dot{e}>0 \cap \dot{e}<d_{2}$, then $\lambda_{1}=\Delta_{1}, \lambda_{2}=\Delta_{2}$;

Rule 7 If $e>0 \cap e<d_{1} \cap \dot{e}<0 \cap \dot{e}>-d_{2}$, then $\lambda_{1}=\Delta_{1}, \lambda_{2}=\Delta_{2}$;

Rule 8 If $e>0 \cap e<d_{1} \cap \dot{e}<-d_{2}$, then $\lambda_{1}=0, \lambda_{2}=\Delta_{2}$;

Rule 9 If $e<0 \cap e>-d_{1} \cap \dot{e}>d_{2}$, then $\lambda_{1}=0, \lambda_{2}=\Delta_{2}$;

Rule 10 If $e<0 \cap e>-d_{1} \cap \dot{e}>0 \cap \dot{e}<d_{2}$, then $\lambda_{1}=\Delta_{1}, \lambda_{2}=\Delta_{2}$;

Rule 11 If $e<0 \cap e>-d_{1} \cap \dot{e}<0 \cap \dot{e}>-d_{2}$, then $\lambda_{1}=\Delta_{1}, \lambda_{2}=\Delta_{2}$;

Rule 12 If $e<0 \cap e>-d_{1} \cap \dot{e}<-d_{2}$, then $\lambda_{1}=\Delta_{1}, \lambda_{2}=0$;

Rule 13 If $e<-d_{1} \cap \dot{e}>d_{2}$, then $\lambda_{1}=0, \lambda_{2}=-\Delta_{2}$;

Rule 14 If $e<-d_{1} \cap e>0 \cap \dot{e}<d_{2}$, then $\lambda_{1}=\Delta_{1}, \lambda_{2}=\Delta_{2}$;

Rule 15 If $e<-d_{1} \cap e<0 \cap \dot{e}>-d_{2}$, then $\lambda_{1}=0, \lambda_{2}=\Delta_{2}$;

Rule 16 If $e<-d_{1} \cap \dot{e}<-d_{2}$, then $\lambda_{1}=0, \lambda_{2}=0$ 。 where $e, \dot{e}$ are the heading deviation and its differentiation of the USV at the current time, $d_{1} 、 d_{2}$ are the reference threshold set according to the variation of $e 、 \dot{e}, \lambda_{1} 、 \lambda_{2}$ are dynamic correction factors of control parameters $k_{1}$ 、 $k_{2}, \Delta_{1} 、 \Delta_{2}$ are the increments corresponding to $k_{1} 、 k_{2}$ , which need to be selected according to the actual situation. Assuming that $k_{1}(t) 、 k_{2}(t) 、 k_{1}(t-1) 、 k_{2}(t-1)$ are the control parameters at time $t$ and $t-1$, then the online correction algorithms for $k_{1} 、 k_{2}$ can be described as follows:

$$
\left\{\begin{array}{l}
k_{1}(t)=k_{1}(t-1)+\lambda_{1} \\
k_{2}(t)=k_{2}(t-1)+\lambda_{2}
\end{array}\right.
$$

where $k_{1}(0) 、 k_{2}(0)$ are the initial values of $k_{1} 、 k_{2}$, and can be selected according to control system characteristics and practical experience.

\section{EXPERIMENTS AND RESULTS}

Based on the designed adaptive expert S-PID control algorithm for heading control system, several pool experiments and lake experiments are carried out with SL micro-USV. It is important to note during the experiments that the compass should be placed as far as possible from the wireless communication module and GPS module, because their signal transmission would interfere with it. The wireless communication module and the STM32-ARM control core board module need to be powered separately, while the GPS module could be powered by STM32-ARM control core board. If the wireless communication module is powered by STM32-ARM control core board, the communication module would interfer the GPS signal and the output of the SMT32-ARM core control board.

\section{POOL EXPERIMENTS}

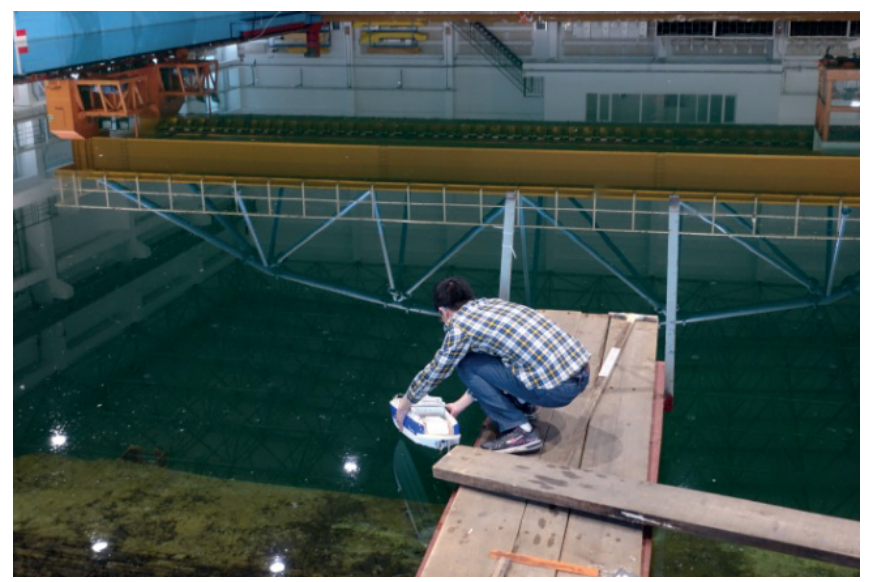

(a) launching of USV 


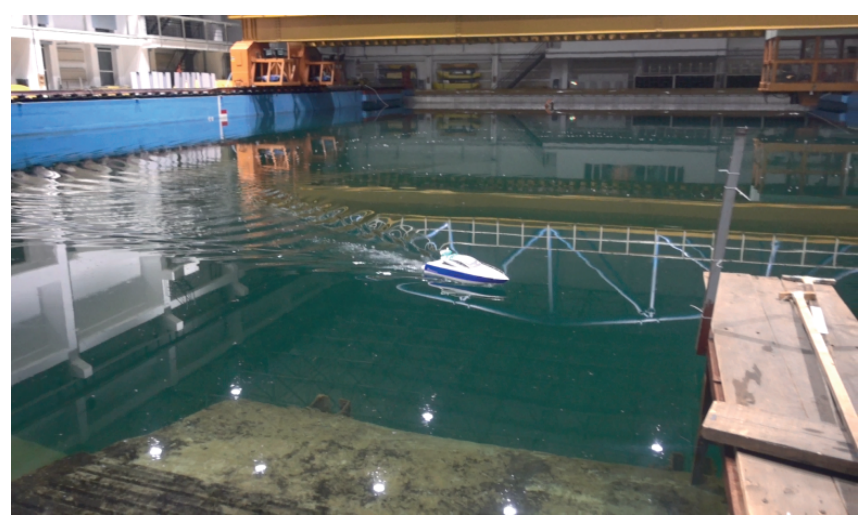

(b) pool experiment of USV

Fig.8. Pool experiment scene of USV

A series of pool experiments are carried out firstly to verify the reliability of the designed control system and the superiority of the controller, the experimental scenario is shown above in Figure 8 and the heading control experiment results are shown in Figure 9.

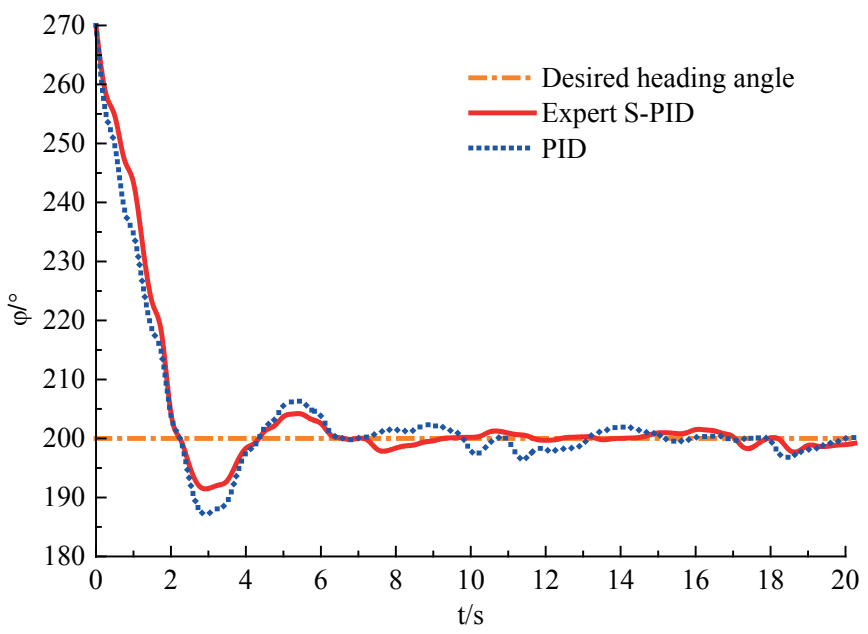

(a) $270 \mathrm{deg}-200 \mathrm{deg}$ heading control results

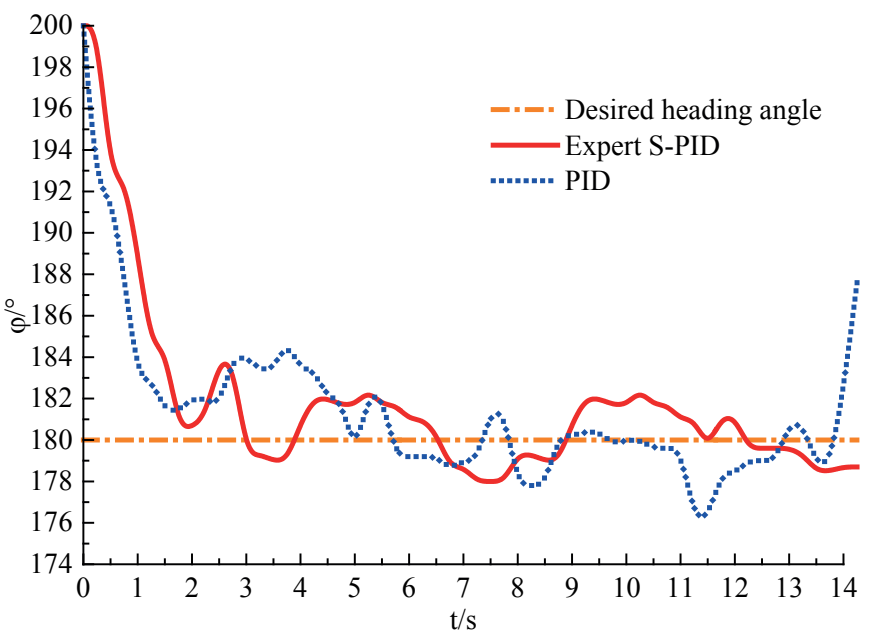

(b) $200 \mathrm{deg}-180 \mathrm{deg}$ heading control results

Fig.9. Heading control pool experiment results of USV
Figure 8 shows the experimental scenario in which the authors carried out the heading control experiments by the SL micro-USV in the pool. The pool is 50 meters long, 20 meters wide and 10 meters deep. Figure 9(a) shows heading control results of SL micro-USV from 270deg to $200 \mathrm{deg}$ and Figure 9(b) shows results from $200 \mathrm{deg}$ to $180 \mathrm{deg}$, where the yellow dot dash line represents the desired heading angle of SL micro-USV, the red solid line represents the heading control results of the USV by expert S-PID algorithm proposed in this paper, the blue dot line represents the heading control results of the USV by traditional PID algorithm. All these markings are the same in other figures. As it can be seen from Figure 9, (a) and (b), the SL USV's average heading control error by traditional PID control algorithm is about 5-6 deg while it is 2-3 deg by the adaptive expert S-PID control algorithm proposed in this paper. In addition, compared with the results obtained by traditional PID control algorithm, the overshoot of the results obtained by adaptive expert S-PID control algorithm is small and the stability is better.

\section{LAKE EXPERIMENTS}

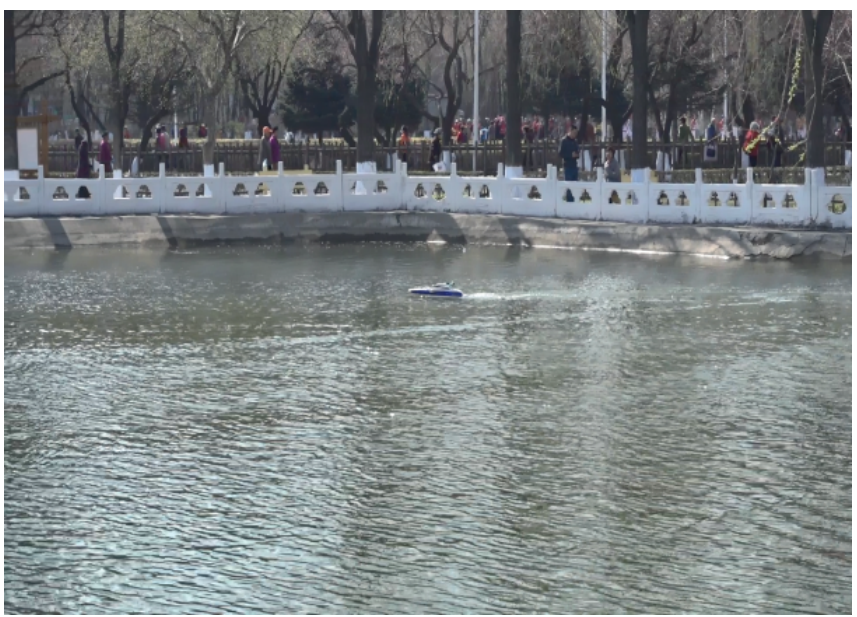

(a) Lake experiment of USV

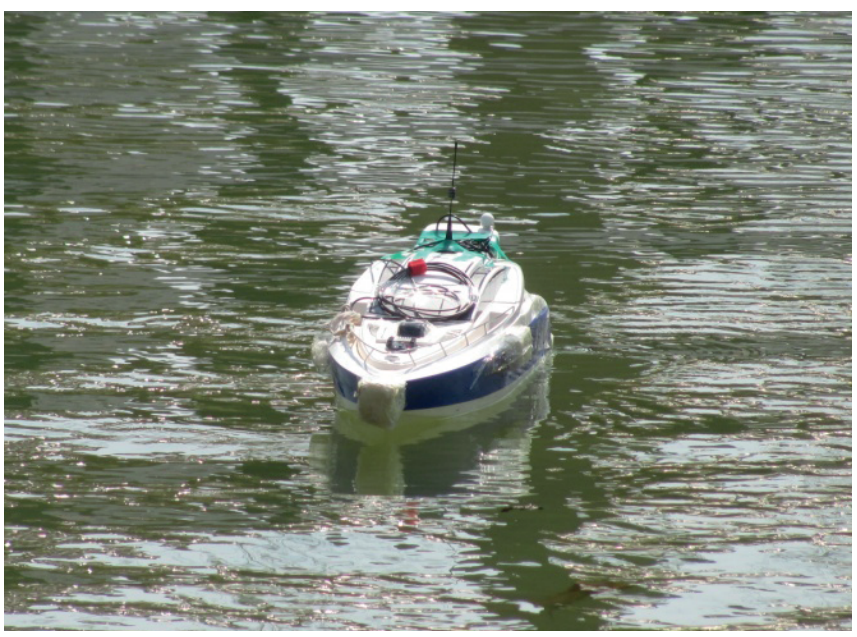

(b) USV on the lake

Fig.10. Lake experiment scene of USV 
Secondly, a series of lake experiments are carried out to verify the reliability of the designed control system and the superiority of the controller. The experimental scenario is shown above in figure 10 and the heading control experiment results are shown in figure 11.

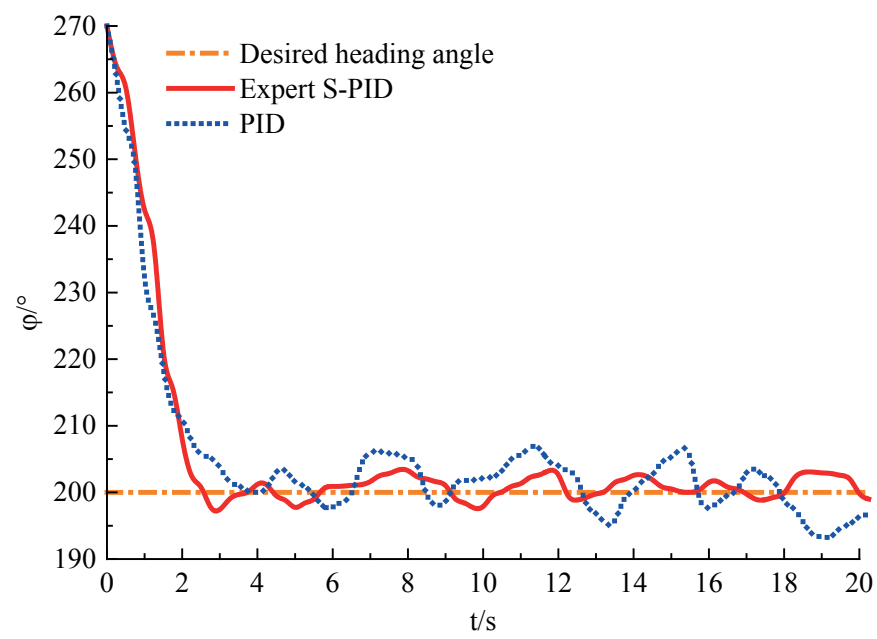

(a) 270deg-200deg heading control results

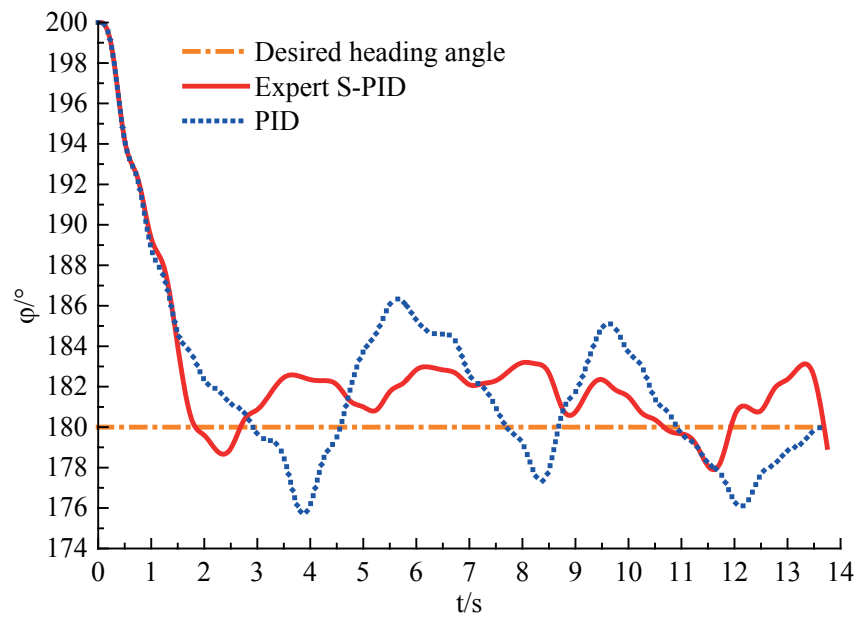

(b) $200 \mathrm{deg}-180 \mathrm{deg}$ heading control results

Fig.11. Heading control lake experiment results of USV

In order to further verify the reliability and validity of the proposed adaptive expert S-PID algorithm for the SL USV's heading control system, outdoor lake experiments are carried out. There are weak disturbances caused by wind and waves on the lake, which can be seen in figure 10. Figure 11(a) shows the heading control results of SL USV from $270 \mathrm{deg}$ to $200 \mathrm{deg}$ and figure 11(b) shows the results from $200 \mathrm{deg}$ to $180 \mathrm{deg}$, which are obtained in the lake experiments. As it can be seen from figure 11, (a) and (b), the SL USV's average heading control error by traditional PID control algorithm is about 6-7 deg while it is 3-4 deg by the adaptive expert S-PID control algorithm proposed in this paper. In addition, compared with the results obtained by traditional PID control algorithm, the overshoot of the results obtained by the adaptive expert $\mathrm{S}$-PID control algorithm is smaller and the stability is better.

\section{CONCLUSION}

This paper proposed an adaptive expert S-PID control algorithm for SL USV's heading control system. The motion control system for the micro-USV is designed based on STM32-ARM, and the PC monitoring system is developed based on Labwindows/CVI. The hardware system and software system for the SL USV proposed in this paper can provide reliable reference and technical support for the development of other micro USV systems. Based on the artificial parameter adjustment experience accumulated by the authors research group, expert control technology is utilized to design an intelligent coordinator for the adaptive adjustment of the $S$ algorithm's control parameter, and then the traditional PID control algorithm is fused. The expert intelligent coordination optimization algorithm can optimize the $S$ plane algorithm's control parameters in real time, which realizes the adaptive adjustment of the control parameters and allows to avoid the complicated manual adjustment process. A large number of pool experiments and lake experiments are carried out, to verify the effectiveness and reliability of the designed motion control system and the heading control algorithm proposed in this paper. Experiment results show that the proposed adaptive expert S-PID control algorithm has s 2-3deg improvement over the traditional PID control algorithm in heading control of SL micro-USV. It is also noted that the control stability and the reliability are both better.

\section{ACKNOWLEDGEMENTS}

This work was supported by the National Natural Science Foundation of China (Grant Nos. 51409054, 51409059, 51409061, 51509057 and 51579022) and the National High Technology Research and Development Program 863 of PR China (No. 2014AA09A509).

\section{REFERENCES}

1. Woo J., Kim N. Vision based target motion analysis and collision avoidance of unmanned surface vehicles. Proceedings of the Institution of Mechanical Engineers Part M-Journal of Engineering for the Maritime Environment, 2016, 230(4): 566-578.

2. Dong Z.P., Wan L., Liu T., et al. Horizontal plane trajectory tracking control of an underactuated unmanned marine vehicle in the presence of ocean currents. International Journal of Advanced Robotic Systems, 2016, 13:83, 1-14.

3. Nuss A., Blcakburn T., Garstenauer A. Toward resilient unmanned maritime systems (UMS). Naval Engineers Journal, 2016, 128(2): 65-71.

4. Przyborski M. Information about dynamics of the sea surface as a means to improve safety of the unmanned vessel at sea. Polish Maritime Research, 2016, 23(4): 3-7. 
5. Caccia M., Bono R., Bruzzon G., et al. Sampling sea surfaces with SESAMO. IEEE Robotics and Automation Magazine, 2015, 12(3): 95-105.

6. Do K D, Pan J. Robust path-following of underactuated ships: theory and experiments on a model ship. Ocean Engineering, 2006, 33(10): 1354-1372

7. Sohn S I, Oh J H, Lee Y S, et al. Design of a fuel-cell-powered catamaran-type unmanned surface vehicle. IEEE Journal of Oceanic Engineering, 2015, 40(2): 388-396

8. Wu G X, Sun H B, Zou J, et al. The basic motion control strategy for the water-jet-propelled USV. In the proceedings of 2009 IEEE International Conference on Mechatronics and Automation, Changchun, China, August 9-12, 2009, IEEE, pp: 611-616.

9. Li Z, Sun J. Disturbance compensating model predictive control with application to ship heading control. IEEE Transactions on Control Systems Technology, 2012, 20(1): 257-265.

10. Sonnenburg C R, Woolsey C A. Modeling, identification and control of an unmanned surface vehicle. Journal of Field Robotics, 2013, 30(3): 371-398.

11. Kurowski M, Haghani A, Koschorrek P, et al. Guidance, navigation and control of unmanned surface vehicles. AT-Automatisierungstechnik, 2015, 63(5): 355-367.

12. Nad D, Miskovic N, Mandic F. Navigation, guidance and control of an overactuated marine surface vehicle. Annual Reviews in Control, 2014, 40: 172-181.

13. Wang N., Lv S.L., Liu Z.Z. Global finite time heading control of surface vehicles. Neurocomputing, 2016, 175: 662-666.

14. Veksler A., Johansen T. A., Borrelli F., et al. Dynamic position with model predictive control. IEEE Transactions on Control Systems Technology, 2016, 24(4): 1340-1353.

15. Wang Y. L., Han Q. L. Network based heading control and rudder oscillation reduction for unmanned surface vehicles. IEEE Transactions on Control Systems Technology, 2016: $1-12$.

16. Dong Z. P., Wan L., Li Y. M., et al. Trajectory tracking control of underactuated USV based on modified backstepping. International Journal of Naval Architecture and Ocean Engineering, 2015, 7(5): 817-832.

17. Alfi A., Shokrzadeh A., Asadi M. Reliability analysis of H-infinity control for a container ship in way-point tracking. Applied Ocean Research, 2015, 52: 309-316.

\section{CONTACT WITH THE AUTHORS}

\author{
Zaopeng Dong \\ e-mail:naval_architecture@163.com \\ School of Transportation \\ Wuhan University of Technology \\ 122 Luoshi Road \\ 430070 Wuhan
}

China 